\title{
Caracterización clínica y epidemiológica de infección asociada a atención en salud por virus influenza en pacientes críticos
}

\author{
Valentina Gutiérrez¹, Jaime Cerda², Nicole Le Corre ${ }^{3}$, Rafael Medina ${ }^{4}$ y Marcela Ferrés ${ }^{3}$
}

${ }^{1}$ Residente de Enfermedades Infecciosas del Niño, Departamento de Enfermedades Infecciosas e Inmunología Pediátrica, Pontificia Universidad Católica de Chile, Santiago, Chile. 2Departamento de Salud Pública, Pontificia Universidad Católica de Chile, Santiago, Chile. ${ }^{3}$ Departamento de Enfermedades Infecciosas e Inmunología Pediátrica, Laboratorio de Infectología y Virología Molecular, Pontificia Universidad Católica de Chile, Santiago, Chile ${ }^{4}$ Departamento de Enfermedades

Infecciosas e Inmunología Pediátrica, Laboratorio de Virología Molecular, Pontificia Universidad Católica de Chile, Santiago, Chile.

$N^{\circ}$ de Proyecto: 170316007 , Proyecto investigación de subespecialidad enfermedades infecciosas del niño.

Proyecto Anillo ACT 1408,

Proyecto Fondecyt 1161791 No hay conflictos de interés que declarar.

Recibido: 19 de junio de 2018 Aceptado: 28 de marzo de 2019

Correspondencia a: Marcela Ferrés Garrido mferres@med.puc.c

\section{Introducción}

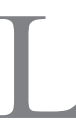

as infecciones respiratorias virales adquiridas en la atención de salud aumentan la morbilidad, mortalidad y costos en salud. Se estima que alrededor del $20 \%$ de las personas con neumonía asociada a atención en salud presenta una infección viral ${ }^{1}$.

En Chile, durante el año 2017 se notificó un total de 3.505 casos de infecciones respiratorias por virus influenza ${ }^{2}$. Del total de las causas respiratorias, influenza y neumonía presentan una tasa de mortalidad que se ha mantenido en 20 por 100.000 habitantes (2001-2010), causando en promedio 3.500 muertes/año en los últimos 10 años ${ }^{3}$. Entre los virus respiratorios, datos de Estados Unidos de América señalan que el virus influenza infecta aproximadamente a $5-20 \%$ de la población, resultando en 226.000 hospitalizaciones y 36.000 muertes. Su transmisión ha sido descrita en ambientes hospitalarios siendo el personal de salud, uno de los grupos identificados como casos índice de brotes nosocomiales ${ }^{4}$.

El virus de influenza es un virus ARN, segmentado, envuelto, lábil a los solventes lipídicos. El período de incubación del virus influenza es de uno a cuatro días, con un promedio de dos, y la transmisión puede ocurrir 24 h previo al inicio de los síntomas. La excreción viral alcanza su máximo al tercer día de sintomatología y disminuye a los siete días, pero puede prolongarse en niños e inmunocomprometidos por 10 días o más ${ }^{5,6}$. La transmisión viral puede ocurrir a través de gotitas; sin embargo, en la población infantil la transmisión por contacto directo se ha evidenciado por la recuperación del virus desde fómites en 23 a $53 \%$, que son utilizados en jardines infantiles durante la temporada del brote estacional ${ }^{7}$. Se ha evidenciado la viabilidad del virus influenza en superficies desde dos días a dos semanas, siendo más prolongada en superficies de acero inoxidable ${ }^{8}$. La transmisión por la vía aérea es excepcional; sin embargo, es importante considerarla en el manejo de pacientes inmunocomprometidos.

Los pacientes con mayor riesgo de adquirir una infección grave por virus influenza son los pacientes en edades extremas, obesos mórbidos (IMC $\geq 40$ ), en unidades de cuidados intensivos (UCI), con inmunodeficiencia primaria y secundaria, con patologías crónicas (cardiopulmonares, diabetes mellitus), entre otros ${ }^{9,10}$. Estudios comparativos entre neumonía adquirida en el hospital versus en la comunidad, han evidenciado que los pacientes que cursan con infección intrahospitalaria son de mayor edad, con terapia inmunosupresora, receptores de 
trasplantes de órganos sólidos, malignidades hematológicas y con baja cobertura de vacunación. La frecuencia de neumonía nosocomial por virus influenza en estos estudios fue de $24-26 \%{ }^{11,12}$. Huzly y cols., reportaron letalidad de $9 \%$ en pacientes con neumonía intrahospitalaria por virus influenza ${ }^{11}$.

En un servicio de UCI neonatal, Bennet y cols. ${ }^{13}$, estudiaron la incidencia de infecciones virales respiratorias en niños prematuros asintomáticos y hospitalizados desde su nacimiento. Luego de dos meses, 52\% resultó positivo para uno o más virus respiratorios, lo que se asoció a mayor estadía hospitalaria, soporte ventilatorio y displasia broncopulmonar.

En el Hospital Clínico Red de Salud UC Christus (RS$\mathrm{UCCH}$ ), a través de la vigilancia de infecciones virales respiratorias asociadas a atención en salud, se estimó que durante el año 2014, del total de pacientes hospitalizados y con infecciones virales respiratorias, $15 \%$ de éstas fueron adquiridas durante la atención de salud. El virus influenza ocupó el primer lugar y más de $50 \%$ de éstas ocurrieron en pacientes hospitalizados en unidades críticas (Ferrés y cols., data no publicada). A pesar que influenza es la única de las infecciones virales respiratorias prevenibles con vacuna y una terapia específica, los casos de IAAS siguen ocurriendo. El objetivo primario de este proyecto fue caracterizar los aspectos clínicos, epidemiológicos y microbiológicos de los pacientes con IAAS por influenza hospitalizados en unidades de pacientes críticos (UPC: UCI y Cuidados Intermedio-CI) y Cuidados Especiales (CE). Como objetivos secundarios se evaluó el cumplimiento a las precauciones adicionales a las precauciones estándar (PAPE) y la adherencia a la vacunación contra influenza.

\section{Material y Métodos}

\section{Tipo de estudio}

Descriptivo.

\section{Población}

Pacientes hospitalizados en UPC y CE con IAAS influenza entre los años 2014 y 2017 en el Hospital Clínico RS-UCCH. IAAS por influenza A o B. Se definió como el inicio de síntomas (respiratorios, fiebre, cefalea y/o mialgias) con un test de reacción de polimerasa en cadena por transcriptasa reversa (RPC-TR) positiva a las 48 h o más de ingreso hospitalario, sin sintomatología respiratoria previa o con un estudio de influenza negativo al ingreso. La RPC se realizó por panel respiratorio Seeplex RV15 ${ }^{\circledR}$ durante los años 2014-2015 y por NxTAG Luminex ${ }^{\circledR}$ en 2016-2017. Cuando la muestra respiratoria estuvo disponible se realizó cultivo viral para virus influenza en células MDCK.
Entre los exámenes disponibles para el diagnóstico de virus influenza existen: cultivo, inmunofluorescencia, test rápidos de influenza y $\mathrm{RPC}-\mathrm{TR}$. El cultivo es altamente específico y sensible, pero el resultado se demora entre 48 a 72 h por lo que no es útil para tomar decisiones clínicas rápidas. El test rápido de influenza presenta alta especificidad, $\geq 95 \%$ para ambos virus, pero baja sensibilidad (78,7\% para virus A y 74,3\% para virus B). En nuestra institución se utiliza RPC-TR como técnica de elección en los pacientes hospitalizados por su alta sensibilidad y especificidad, $\geq 95 \%$ para ambos virus. Cabe mencionar que la sensibilidad depende de la celularidad de la muestra, los días de evolución del cuadro clínico y el tipo de muestra (el aspirado nasofaríngeo es la muestra más sensible $)^{14-16}$.

Las unidades hospitalarias consideradas como de atención del paciente crítico se dividieron en tres grupos: Cuidados especiales, corresponde al servicio con pacientes crónicos, de edad avanzada o enfermos terminales que requieren monitoreo constante y mayor atención de enfermería (concentra pacientes que requieren apoyo de medicina interna y cardiovascular), Unidades de Intermedio (Neonatología, Pediátrica, Médica, Neurológica) y Unidades de Cuidados Intensivos (Neonatal, Pediátrica, Médica, Quirúrgica, Coronaria).

Los casos de IAAS fueron identificados a partir de los registros de RPC-TR positivas para virus influenza procesadas en el Laboratorio de Infectología y Virología Molecular de la RS-UCCH. Una vez que los pacientes fueron identificados y cumplieron con los criterios de inclusión de IAAS, se revisaron las fichas médicas y se verificó la pertenencia de ellos al proyecto Fondecyt 1161791 para registrar datos de respuesta inmunológica específica al virus de la influenza (título de inhibición de hemoaglutinación). La caracterización de cada paciente se realizó midiendo las siguientes variables: edad, patología de base del paciente, enfermedades crónicas en tratamiento e inmunosupresión. Los pacientes inmunocomprometidos se clasificaron en receptores de trasplante de órganos sólidos (TOS), receptores de trasplante de precursores hematopoyéticos (TPH), con enfermedad autoinmune, uso prolongado de corticosteroides $(>1 \mathrm{mg} / \mathrm{kg} /$ día por más de 7 días) e infectados por virus de inmunodeficiencia humana (VIH), entre otros.

Con respecto a la expresión clínica, se registró si hubo descompensación de una enfermedad de base y/o respiratoria (inicio o aumento de requerimientos de oxígeno, uso de ventilación mecánica, traslado a unidades de mayor complejidad) y presencia de co-infección respiratoria.

Se registró el uso de PAPE, prescripción de antivirales, antimicrobianos, uso de otros recursos hospitalarios (oxigenoterapia, días de ventilación mecánica, días de hospitalización en UPC y totales) y se consignó la letalidad.

Además, se registró el estado de vacunación contra 
virus influenza del año correspondiente para cada paciente, accediendo al Registro Nacional de Inmunizaciones del Ministerio de Salud (MINSAL) disponible desde el año 2010 .

\section{Definiciones operacionales}

Infección asociada a la atención de salud (IAAS): Infección adquirida por los pacientes en un establecimiento de salud (abierto o cerrado) $48 \mathrm{~h}$ luego de su ingreso o atención, la que no se encontraba presente al momento del ingreso o consulta, excepto si la infección estuviese relacionada a admisiones previas en la misma institución.

Neumonía asociada a la atención de salud: Neumonía que ocurriese $\geq 48 \mathrm{~h}$ después del ingreso hospitalario y que no pareciera haber estado incubándose al momento de la admisión.

Precauciones adicionales a las precauciones estándar (PAPE): Específicamente en virus influenza en adultos se utilizan precaución gotitas (uso de mascarilla), en pediatría se adiciona contacto (uso de pechera y guantes).

Días de hospitalización por influenza: Número de días de hospitalización posterior al inicio de síntomas, con examen positivo para este virus.

Co-infección respiratoria: Identificación de otro agente (virus, bacteria y/u hongo) en el período del diagnóstico de influenza $( \pm 72 \mathrm{~h})$.

Descompensación de patología de base: Reagudización de enfermedad renal crónica o de insuficiencia cardiaca.

Descompensación respiratoria: Requerimientos de oxígeno o aumento de los requerimientos basales. Requerir apoyo con ventilación mecánica no invasiva o invasiva.

Atribución a influenza: Paciente cursando con influenza sin otro diagnóstico presuntivo.

\section{Análisis estadístico}

En este estudio se utilizó estadística descriptiva. En aquellas situaciones en que existían variables a comparar en dos grupos se utilizó prueba $\chi^{2}$ en programa Statistical Package for the Social Sciences (SPSS). Se consideró estadísticamente significativo un valor $\mathrm{p} \leq 0,05$.

\section{Aspectos éticos del Proyecto}

El estudio fue revisado y aprobado por el Comité de Ética en Investigación de la Escuela de Medicina Pontificia Universidad Católica bajo el protocolo N 170316007 que cuenta con dispensa de consentimiento informado y autoriza el uso de información retrospectiva basado en revisión de fichas clínicas.

\section{Resultados}

Durante los años 2014 a 2017, un total de 22 casos de IAAS por influenza cumplió con los criterios de inclusión en los servicios mencionados correspondientes a 187 camas.

El 54\% (12/22) de los casos fueron diagnosticados durante el año 2014, siguiendo en frecuencia el 2017 (18\%) y, en último lugar, los años 2015 y 2016, ambos con $14 \%$. La IAAS más frecuente fue por virus influenza A con $77 \%$. Los casos se concentraron en invierno, 91\% (20/22), sólo dos casos ocurrieron en primavera, ambos por influenza $B$.

\section{Características de la población estudiada}

La mediana de edad fue de 74 años (rango: 4 meses a 93 años), hubo sólo tres casos pediátricos, siendo $86 \%$ $(19 / 22)$ mayores de 18 años. Predominó el sexo femenino con $64 \%(14 / 22)$ de los casos.

La infección fue adquirida entre los días 3 y 126 de hospitalización (mediana 13 días). Al momento del diagnóstico, siete (32\%) estaban internados en UCI y seis (27\%) en Cuidados Intermedios. Del total de pacientes, sólo tres (14\%) habían ingresado por problemas pulmonares (Tabla 1).

Ochenta y seis por ciento $(19 / 22)$ de los pacientes presentaba alguna co-morbilidad siendo la más frecuente hipertensión arterial (HTA) $(13 / 22,59 \%)$. Dieciocho por ciento (4/22) de los pacientes eran inmunocomprometidos, incluyendo pacientes receptores de TOS, TPH, usuario de corticosteroides y pacientes con infección por $\mathrm{VIH}$ de reciente diagnóstico (Tabla 2).

Hubo co-infección respiratoria en $27 \%(6 / 22)$ de los pacientes con IAAS por influenza; $83 \%(5 / 6)$ con

Tabla 1. Motivo de ingreso de los 22 pacientes diagnosticados con IAAS por influenza

\begin{tabular}{ll}
\hline Motivo de ingreso & $\mathbf{n ~ ( \% )}$ \\
UPC $\mathrm{n}=13$ & \\
Compromiso SNC y periférico & $6(46)$ \\
Problemas pulmonares & $2(15)$ \\
Otras causas* & $5(38)$ \\
\hline Cuidados especiales $n=9$ & \\
Compromiso SNC & $3(33)$ \\
Fractura de cadera & $2(22)$ \\
Problema pulmonar & $1(11)$ \\
Otras causas* & $3(33)$ \\
\hline
\end{tabular}

*Politrauma, rabdomiólisis, pancreatitis, falla renal, sepsis, hernia abdominal, Infección del tracto urinario y trombosis venosa profunda. 
al menos otro virus identificado (la totalidad de las coinfecciones se describen en la Tabla 3). Al comparar los pacientes con co-infección y sin co-infección, todos los pacientes con co-infección utilizaron antimicrobianos, requirieron más días de oxigenoterapia y de hospitalización posterior al diagnóstico de influenza; sin embargo, exceptuando hospitalización por más de 14 días, estas diferencias no fueron estadísticamente significativas (Tabla 4).

\section{Uso de recursos hospitalarios posterior al diagnóstico de IAAS influenza}

El 54\% (12/22) de los pacientes utilizó oxígeno mientras cursaron con influenza. Dieciocho por ciento (4/22) estaba conectado a ventilación mecánica por otras indicaciones cuando se detectó la infección. Del total, 72\% (16/22) evolucionaron en UPC, destacando que tres fueron trasladados mientras cursaban con la enfermedad, uno de ellos a UCI (por infarto) y dos a la unidad de intermedio por descompensación respiratoria (tromboembolismo pulmonar y neumonía asociada a atención en salud) concomitante con influenza. La mediana de hospitalización en UCI post diagnóstico de influenza fue de 12 días (rango 4-39), en Intermedio cuatro días (rango 1-9) y en Cuidados Especiales cuatro días (rango 3-29 días). La mediana del total de días de hospitalización para estos pacientes fue de 30 días (7-134) y post diagnóstico de influenza 10 días (3-43 días).
Tabla 2. Características demográficas y clínicas de los 22 pacientes con IAAS por influenza

\begin{tabular}{|c|c|}
\hline Características & \\
\hline Edad, mediana años (rango) & $74(4 m-93 a)$ \\
\hline Sexo, n, (\%) & \\
\hline Femenino & $14(64)$ \\
\hline Adquisición de IAAS, mediana días, (rango) & $13(3-126)$ \\
\hline Procedencia, n (\%) & \\
\hline UCl & $7(32)$ \\
\hline Intermedio & $6(27)$ \\
\hline CE & $9(41)$ \\
\hline Comorbilidad, n (\%) & \\
\hline HTA & $13(59)$ \\
\hline ERC & $6(27)$ \\
\hline Obesidad & $4(18)$ \\
\hline ICC & $3(14)$ \\
\hline AVE & $3(14)$ \\
\hline Inmunosupresión & $4(18)$ \\
\hline Coinfección respiratoria, n (\%) & $6(27)$ \\
\hline Descompensación, n (\%) & $11(50)$ \\
\hline Vacunación, n (\%) & $9(41)$ \\
\hline
\end{tabular}

UCl: Unidad de cuidados intensivos; CE: Cuidados especiales; HTA: Hipertensión arterial; ERC: Enfermedad renal crónica; ICC: Insuficiencia cardiaca congestiva; AVE: Accidente vascular encefálico. Para evaluación de obesidad se contó con medidas antropométricas de 19 pacientes ( 3 pacientes sin datos de peso y/o talla IMC en ficha clínica).
Tabla 3. Descripción de 6 pacientes con influenza y co-infección por otros agentes

\begin{tabular}{cccc}
\hline n paciente & Viral & Bacteriana & Fúngica \\
1 & ParaFLU 1 & & \\
2 & BocaV + RV/EV & Klebsiella pneumoniae & \\
3 & $\mathrm{RV}$ & $\begin{array}{c}\text { SAMR + Stenotrophomona }+ \\
\text { Klebsiella pneumoniae }\end{array}$ & \\
4 & $\mathrm{CMV}$ & Pneumocystis jiroveci \\
\hline 5 & $\mathrm{CMV}$ & Nocardia & \\
\hline 6 & & & \\
\hline
\end{tabular}

ParafLU1: Parainfluenza tipo 1; BocaV: Bocavirus; RV: Rinovirus; EV: Enterovirus; CMV: Citomegalovirus.

Tabla 4. Características clínicas de los pacientes con IAAS por influenza con o sin co-infección

\begin{tabular}{lccc}
\hline & $\begin{array}{c}\text { Co-infección } \\
(\mathbf{n}=\mathbf{6})\end{array}$ & $\begin{array}{c}\text { Sin co-infección } \\
(\mathbf{n}=\mathbf{1 6})\end{array}$ & Valor $\mathbf{p}$ \\
Edad, años (rango) & $81(6,3-87)$ & $74(0,3-93)$ & 0,90 \\
\hline $\begin{array}{l}\text { Procedencia, } \mathrm{n}(\%) \\
\text { UCI }\end{array}$ & $4(66.6)$ & $3(18)$ & 0,09 \\
Intermedio & $1(16)$ & $5(31)$ & \\
CE & $1(16)$ & $8(58)$ & \\
02 post FLU, días (rango) & $14,5(13-28)$ & $3,5(2-33)$ & 0,14 \\
Hosp, días (rango) & $35,5(10-106)$ & $22(7-134)$ & 0,53 \\
Hosp post FLU, días (rango) & $19,5(5-28)$ & $8,5(3-48)$ & 0,23 \\
Hosp post FLU > 14 días, $n(\%)$ & $5(83)$ & $4(25)$ & 0,02 \\
ATB, n (\%) & $6(100)$ & $9(56)$ & 0,13 \\
ATB por causa resp, $n$ (\%) & $5(83)$ & $6(37,5)$ & 0,07 \\
Mortalidad & $2(33)$ & $2(12)$ & 0,29 \\
\hline
\end{tabular}

Las variables numéricas son expresadas en mediana. Hosp = Hospitalización; FLU = Infección por virus influenza; resp: respiratoria. 


\begin{tabular}{|c|c|c|}
\hline & Vacunados (n 9) & No vacunados (n 6) \\
\hline Edad, mediana años (rango) & $80(68-93)$ & $85,5(72,5-88)$ \\
\hline Sexo femenino, $\mathrm{n}(\%)$ & $6 \quad(66)$ & $4 \quad(66)$ \\
\hline IAAS por influenza A, n (\%) & $7 \quad(77)$ & $5 \quad(83)$ \\
\hline $\begin{array}{c}\text { Comorbilidad, n (\%) } \\
\quad \geq 2 \text {, n (\%) }\end{array}$ & $\begin{array}{rr}9 & (100) \\
8 & (89)\end{array}$ & $\begin{array}{rr}6 & (100) \\
4 & (66)\end{array}$ \\
\hline Co-infección, n (\%) & $2 \quad(22)$ & $2 \quad(33)$ \\
\hline Descompensación, n (\%) & $7 \quad(77)$ & $2 \quad(33)$ \\
\hline Inmunosupresión, n (\%) & (0) & $2 \quad(33)$ \\
\hline Mortalidad, n (\%) & $1 \quad(11)$ & $2 \quad(33)$ \\
\hline
\end{tabular}

\section{Diagnóstico de influenza}

Se infectaron por virus tipo A 77\% (17/22) de los pacientes, presente en todos los años, excepto 2015; la tipificación correspondió a H1N1 en el año 2016 y durante el 2017 predominó H3N2 en 75\% de los casos. Las IAAS por influenza tipo B se presentaron durante los años 2014 y 2015, sin casos reportados durante los años 2016-2017.

En 10 pacientes se realizó cultivo viral, lográndose aislamiento sólo en tres de las muestras que se conservaron congeladas a $(-) 80^{\circ} \mathrm{C}(30 \%)$.

\section{Terapia utilizada}

La mayoría de los pacientes recibió oseltamivir 18/22 ( $82 \%$ ) dentro de las $24 \mathrm{~h}$ del diagnóstico virológico. En los cuatro restantes, no fue posible evidenciar la indicación de oseltamivir en la ficha clínica.

Respecto al uso de antimicrobianos, 68\% (15/22) de los pacientes los utilizaron mientras cursaban con IAAS por influenza, mediana siete días (3 - 22 días). La mayoría de ellos, 73\% (11/15), utilizó antimicrobianos debido al problema respiratorio que los afectaba, mientras que $27 \%$ (4/15) restante tenían indicación por causa no respiratoria. De los once pacientes que utilizaron antimicrobianos por causa respiratoria, ocho tenían clara justificación de mantención de ellos y sólo en dos casos se suspendieron posterior al diagnóstico de infección por influenza. En uno de ellos se decidió su mantención dado la gravedad del paciente a pesar de tener parámetros inflamatorios bajos.

\section{Medidas asociadas al manejo de IAAS y antecedentes de vacunación}

Catorce por ciento $(3 / 22)$ de los pacientes estaban con PAPE al momento del diagnóstico de influenza, tanto por infección asociada a bacterias resistentes o por detección de otros virus respiratorios durante su hospitalización. Con posterioridad al diagnóstico de influenza, el uso de PAPE alcanzó a 86\% (19/22) con una mediana de uso de cinco días (rango 2-34 días).

De los pacientes que adquirieron influenza, sólo $41 \%$ (9/22) había recibido la vacuna estacional de influenza ese año. De los no vacunados, 46\% (6/13) tenían recomendación de vacunación por ser mayores de 65 años, con co-morbilidad asociada y dos de ellos, además, con inmunosupresión. Las características clínicas de los pacientes vacunados y no vacunados se encuentran en la Tabla 5.

Cabe mencionar que uno de los pacientes vacunados que enfermó por influenza A H1N1 no tenía título de anticuerpo protector para A H1N1 $(<1 / 40)$, medidos por inhibición de hemaglutinación, la primera semana del inicio de los síntomas. Sin embargo, sí tenía títulos protectores para influenza A H3N2 ( $\geq 1 / 40)$. No contamos con estos datos para los demás pacientes.

\section{Evolución}

El 50\% (11/12) de los pacientes tuvieron descompensación concomitante con la IAAS por influenza. De éstos, nueve $(82 \%)$ por causa pulmonar, dentro de los cuales sólo cuatro se pudieron atribuir a influenza. Un paciente presentó una descompensación cardíaca (infarto) y uno renal (enfermedad renal aguda). Cuatro pacientes fallecieron (18\%). Todos los fallecidos cursaron con infección por virus tipo A y eran pacientes de mayor edad, sobre 80 años, a excepción de un paciente pediátrico que era receptor de TPH. Todos presentaban al menos dos co-morbilidades, la mitad eran inmunocomprometidos, $75 \%$ estaban hospitalizados en UCI y 50\% con VM. Sólo uno de los fallecidos estaba vacunado contra influenza.

\section{Discusión}

En esta serie, los pacientes con IAAS por influenza fueron fundamentalmente enfermos crónicos, de edad avanzada y que no recibieron vacuna contra influenza, pese a que la mayoría de los no vacunados tenía indicación de acuerdo a las recomendaciones del $\mathrm{MINSAL}^{3}$, lo que concuerda con la literatura científica ${ }^{11,17}$. Cabe destacar que los casos pediátricos con IAAS fueron minoría, y los afectados corresponden al grupo de mayor riesgo (inmunocomprometidos y enfermedad pulmonar crónica).

Durante los años estudiados, las IAAS por virus influenza A coincidieron con los picos estacionales observados en la Región Metropolitana y, en los años 2016-2017, en que se realizó sub-tipificación, hubo concordancia con el subtipo circulante informado por el ISP de Chile. Durante el año 2015 no hubo registro de IAAS por influenza A, probablemente asociado con 
una campaña interna de reforzamiento de medidas de prevención de IAAS basado en educación al personal y familiares. Los casos de influenza B fueron esporádicos. De este análisis podemos rescatar que el aumento de casos de influenza en el país nos debe alertar a tomar medidas aún más estrictas al interior de los hospitales, para evitar la transmisión nosocomial.

Ante el diagnóstico de un paciente hospitalizado con influenza, la conducta médica es evaluar el uso de antivirales y la aparición de eventuales complicaciones. Con respecto al manejo de IAAS, es fundamental enfatizar la adherencia a las recomendaciones de control de infecciones, lavado de manos y uso de $\mathrm{PAPE}^{18,19}$. La mayoría de los pacientes recibió terapia oportuna con oseltamivir dentro de las $24 \mathrm{~h}$ de efectuado el diagnóstico.

Las principales fuentes de contagio, no evaluadas en este trabajo, son el personal de salud y las visitas que el paciente recibe. La transmisión puede ocurrir de forma inadvertida a través de gotitas desde sujetos con o sin síntomas respiratorios que no se auto-excluyen de atender o visitar a estos pacientes. Las visitas representan una fuente de contagio sub-diagnosticada que puede evitarse con educación o prohibición del ingreso hospitalario a los que manifiestan síntomas respiratorios ${ }^{20}$. Se han descrito experiencias exitosas en la prevención de influenza reforzando la educación de la familia en los mecanismos de transmisión y uso de vacunas ${ }^{21,22}$.

La vacunación del personal de salud es otra estrategia importante que puede disminuir la infección respiratoria y eventual transmisión a pacientes vulnerables ${ }^{23}$. Un estudio reciente evidenció disminución de mortalidad por todas las causas en $29 \%$ cuando el personal de salud estaba vacunado $^{24}$. Sin embargo, la cobertura de vacunación contra influenza en esta población ha sido sub-óptima, siendo reportada entre $36-62 \%^{21-23}$. Nuestro estudio no aporta esta información; sin embargo, considerando que la vacunación es voluntaria, debemos educar e incentivar al personal a recibir la vacuna con posterior medición de cobertura y asociarla a resultados futuros.

Desde el punto de vista clínico, en la serie descrita, la mayoría de las descompensaciones fueron respiratorias, si bien éstas no pueden ser atribuidas en su totalidad a la infección por virus influenza ya que los pacientes cursaban con otro cuadro concomitante. Pese a esto, casi un quinto de los casos requirió oxígeno sólo después de adquirir esta infección viral.

Uno de los pacientes presentó un infarto mientras cursaba con influenza. Con respecto a esto, varios estudios sugieren que agentes infecciosos tienen un rol importante en la enfermedad vascular y aterosclerosis ${ }^{28,29}$. Barnes y cols., realizaron una revisión sistemática donde se evidenció que la infección por virus influenza aumentaba en dos veces el riesgo de infarto agudo al miocardio ${ }^{30}$. Nguyen y cols., demostraron mayor mortalidad por causa cardiovascular en pacientes con infección por virus influenza (de 2 a $6 \%$ ), siendo mayor (14\%) para infarto agudo al miocardio ${ }^{31}$. Lenten y cols. ${ }^{32}$, estudiaron ratones infectados con virus influenza A y encontraron que las lipoproteínas de alta densidad perdían su habilidad de oxidar a las LDL, lo que llevaría a mayores eventos ateroscleróticos. Otras teorías tienen relación con la sobre expresión de la vía inflamatoria, daño del endotelio vascular, mayor viscosidad plasmática y cambios en factores de coagulación circulantes que llevarían a la isquemia miocárdica ${ }^{28,31,33}$.

El uso de técnicas moleculares en el diagnóstico de las infecciones respiratorias ha implicado un incremento del espectro etiológico, y la co-detección de más de un virus como también bacterias. En este trabajo encontramos co-infección en $22 \%$ de los pacientes, mientras que en otros se reportan valores entre 10 y $50 \%{ }^{25-27}$. Si bien nuestro total de pacientes es pequeño, el análisis realizado identificó que aquellos con co-infección permanecieron más de 14 días hospitalizados tras el diagnóstico y sin diferencia significativa en mortalidad. En estudios de adultos, la co-infección se ha asociado con peor evolución hospitalaria ${ }^{35-37}$, mientras que en población pediátrica, no se observa mayor diferencia y se discute el rol patogénico de dos o más agentes ${ }^{28-30}$.

Se observó letalidad en $18 \%$, esto es mayor a lo descrito en la literatura médica donde se reporta entre $9 \mathrm{y}$ $11 \%{ }^{11,41}$. Sin embargo, se ha evidenciado mortalidad de $36 \%$ en pacientes hospitalizados en UCI que cursaron con IAAS por influenza A(H1N1)pdm09 ${ }^{12}$. Álvarez-Lerma y cols. ${ }^{12}$, identificaron a los pacientes con mayor riesgo de mortalidad, entre los factores determinantes describen; IAAS por virus $\mathrm{A}(\mathrm{H} 1 \mathrm{~N} 1)$ pdm09, score APACHE II al ingreso a UCI, enfermedad hematológica de base, necesidad de terapia de reemplazo renal y uso de ventilación mecánica. Nuestros pacientes efectivamente presentaron algunos de estos factores de riesgo.

La vacunación es uno de los recursos preventivos, disponible y sin costo, usado en forma programática para proteger, entre otros, a los mayores de 65 años y a aquellos con factores de riesgo de evolución complicada o grave. La cobertura en la población chilena durante los años 2016 y 2017, de acuerdo a información del ISP de Chile, fue de $79 \%$ y $80 \%$, respectivamente ${ }^{42}$. La efectividad de la vacunación en estos años no está disponible; sin embargo, cifras encontradas en los Centers for Disease Control and Prevention (CDC) oscilan entre 20 y $48 \%$ desde el año 2014 al $2017^{43}$.

El porcentaje de vacunación en los pacientes con IAAS por influenza fue menor a $50 \%$. Los valores reportados en otros estudios varían entre 6 y $70 \%{ }^{11,12,41}$. De los vacunados que enfermaron, todos eran mayores de 65 años. Al avanzar la edad, la respuesta inmune inducida por una infección o una inmunización se deteriora, esto 
se conoce como inmunosenescencia. La respuesta innata se encuentra reducida en su capacidad fagocítica y estallido respiratorio. Además, hay defectos en los toll like receptors alterando el reconocimiento antigénico. En la respuesta adaptativa hay menor respuesta de linfocitos $\mathrm{T}$ citotóxicos ${ }^{44}$. Esto trae como consecuencia una menor efectividad por vacuna en estos pacientes, la que ha sido descrita entre 40 y $50 \%{ }^{44-47}$, mientras que en $<65$ años puede alcanzar hasta $80 \%{ }^{46,48}$. Se han estudiado otras opciones para mejorar la respuesta inmune, tales como, mayor dosis de vacuna, uso de adyuvantes y administración intradérmica ${ }^{49-53}$. En el presente trabajo, seis pacientes no vacunados tenían recomendación programática de vacunación por ser mayores de 65 años y tener co-morbilidad asociada ${ }^{3}$. Sin embargo, son IAAS potencialmente evitables, por lo que es de suma importancia cada año educar y fomentar la vacunación de influenza en este grupo etario.

Dentro de las limitaciones de nuestro trabajo está el ser un estudio retrospectivo y de un solo centro, lo que no refleja las regiones geográficas del país ni las diferentes poblaciones ${ }^{54}$. A pesar de que el total de pacientes descritos es pequeño, cabe destacar que los reportes de IAAS por influenza en paciente crítico son escasos. En este sentido, un estudio realizado en unidades de cuidados intensivos, reportó mayor gasto en el tratamiento de pacientes con infecciones intrahospitalarias, en promedio de U\$ 2.958 versus U\$ 1.523 (USD) en quienes no las presentaban. Además de doble estadía en días de UCI y de estadía hospitalaria total, pero sin impacto en mortalidad ${ }^{55}$. En estudios futuros se podría incluir un grupo control para atribuir a influenza las descompensaciones, uso de recursos hospitalarios y cálculo de costos en salud.

Dentro de las fortalezas de esta investigación podemos destacar que se muestra la realidad local y describe el problema actual para futuros estudios. Para mejorar esta caracterización, sugerimos diseñar protocolos multicéntricos y prospectivos, ya que IAAS influenza en paciente crítico es una entidad poco frecuente y aún poco estudiada.

Finalmente, se contribuyó a la caracterización de los pacientes que cursaron con IAAS por influenza en este centro. Esto permitirá la implementación y reforzamiento de medidas preventivas en el cuidado de los pacientes de riesgo, previo al periodo de influenza estacional, con cumplimiento de la vacunación programática, $\mathrm{y}$ al interior de los hospitales con educación y preparación del personal de salud y los visitantes de los pacientes.

\section{Conclusiones}

IAAS por influenza ocurrió en pacientes crónicos, de mayor edad, que ingresaron mayoritariamente por causa no respiratoria y no vacunados. Es primordial en la prevención de IAAS por virus respiratorios, la educación al personal de salud y los familiares, quienes pueden ser la fuente de contagio de estos pacientes, sobre todo en el periodo pico de la influenza estacional. Como otro pilar de la prevención, se debe insistir en mantener una alta cobertura de vacunación en estos pacientes.

Agradecimientos. A Inés Cerón, Infectóloga de Adultos, Pontificia Universidad Católica de Chile, Ana María Contreras y Constanza Martínez Valdebenito, Laboratorio de Infectología y Virología Molecular, Pontificia Universidad Católica de Chile, María Inés Riquelme CPC IAAS, Red de Salud UC Christus.

\section{Resumen}

Introducción: Las infecciones asociadas a atención de salud (IAAS) aumentan la morbilidad y mortalidad. Durante 2014, en Hospital Clínico Red de Salud UC Christus (RS-UCCH) se estimó que $15 \%$ de las infecciones virales respiratorias fueron adquiridas durante la atención de salud, siendo más frecuente el virus influenza. Objetivos: Caracterización clínico-epidemiológica de IAAS por influenza en pacientes hospitalizados en unidades de pacientes críticos (UPC) y cuidados especiales. Material y Métodos: Estudio descriptivo. Se incluyeron pacientes en UPC y cuidados especiales con IAAS influenza entre 2014 y 2017 en RS-UCCH. IAAS por influenza se definió como: inicio de síntomas y/o RPC-TR positiva para virus influenza $\geq 48 \mathrm{~h}$ de ingreso hospitalario, sin sintomatología respiratoria o estudio negativo previo. Resultados: Se identificaron 22 pacientes, edad mediana 74 años. La influenza fue adquirida en promedio al día 13; el $77 \%$ fue por influenza A y el $27 \%$ presentó coinfección respiratoria. Trece (59\%) estaban hospitalizados en UPC, dos (15\%) por problemas pulmonares. El 86\% tenía co-morbilidad y el 50\% descompensación de ella. No estaba vacunado 59\%; la letalidad observada fue $18 \%$. Conclusiones: IAAS por influenza ocurrió en pacientes crónicos, de mayor edad y no vacunados. Es primordial educar en prevención de IAAS y mantener altas coberturas de vacunación. 


\section{Referencias bibliográficas}

1.- Dare R K, Talbot T R. Health care-acquired viral respiratory diseases. Infect Dis Clin North Am 2016; 30(4): 1053-70. doi: 10.1016/j. idc.2016.07.004.

2.- Instituto de Salud. Informe circulación virus respiratorios SE47 28-11-2017. pdf [Internet]. [citado 1 de diciembre de 2017]. Disponible en: http://www.ispch. $\mathrm{cl} /$ sites/default/files/documento/2017/11/ Informe $\% 20$ circulaci $\% \mathrm{C} 3 \% \mathrm{~B} 3 \mathrm{n} \% 20$ virus $\% 20$ respiratorios\%20SE47\%2028-11-2017.pdf

3.- Ministerio de Salud Chile. Guía Práctica Clínica. Prevención, Diagnóstico y Manejo Clínico de Casos de influenza. Abril 2014. https://www.minsal.cl/sites/default/files/files/ GUIA\%20CLINICA\%20INFLUENZA \%20 2014_imprimir.pdf.

4.- Talbot T R, Bradley S F, Cosgrove S E, Ruef C, Siegel J D, Weber D J. Influenza vaccination of healthcare workers and vaccine allocation for healthcare workers during vaccine shortages. Infect Control Hosp Epidemiol 2005; 26 (11): 882-90. doi: $10.1086 / 502512$.

5.- Committee on Infectious Diseases, American Academy of Pediatrics. Red Book: 2015 Report of the Committee on Infectious Diseases. Elk Grove Village, IL: American Academy of Pediatrics; 2015.

6.- Lehners N, Tabatabai J, Prifert C, Wedde M, Puthenparambil J, Weissbrich B, et al. Long-term shedding of influenza virus, parainfluenza virus, respiratory syncytial virus and nosocomial Epidemiology in patients with hematological disorders. PLOS ONE 2016; 11 (2): e0148258. doi: 10.1371/journal. pone. 0148258

7.- Boone S, Gerba C. The occurrence of influenza A virus on household and day care center fomites. J Infect 2005; 51(2):103-9. doi: 10.1016/j.jinf.2004.09.011.

8.- Thompson K-A, Bennett AM. Persistence of influenza on surfaces. J Hosp Infect 2017; 95 (2): 194-9. doi: 10.1016/j.jhin.2016.12.003.

9.- Committee on Infectious Diseases. Recommendations for prevention and control of influenza in children, 2014-2015. Pediatrics 2014; 134: e1503-e1519. https://pediatrics. aappublications.org/content/pediatrics/134/5/ e1503.full.pdf.

10.- Baek J H, Seo Y B, Choi W S, Kee S Y, Jeong H W, Lee H Y, et al. Guideline on the prevention and control of seasonal influenza in healthcare setting. Korean J Intern Med 2014; 29(2): 265. doi: 10.3904/kjim.2014.29.2.265.

11.- Huzly D, Kurz S, Ebner W, Dettenkofer M, Panning M. Characterisation of nosocomial and community-acquired influenza in a large university hospital during two consecutive influenza seasons. J Clin Virol 2015; 73: 47-51. doi: 10.1016/j.jcv.2015.10.016.
12.- Álvarez-Lerma F, Marín-Corral J, Vilà C, Masclans J R, Loeches I M, Barbadillo S, et al. Characteristics of patients with hospitalacquired influenza A (H1N1)pdm09 virus admitted to the intensive care unit. J Hosp Infect 2017; 95 (2): 200-6. doi: 10.1016/j. jhin.2016.12.017.

13.- Bennett N J, Tabarani C M, Bartholoma N M, Wang D, Huang D, Riddell S W, et al. Unrecognized viral respiratory tract infections in premature infants during their birth hospitalization: a prospective surveillance study in two neonatal intensive care units. J Pediatr 2012; 161 (5): 814-8.e3. doi: 10.1016/j. jpeds.2012.05.001.

14.- Peaper D R, Landry M L. Rapid diagnosis of influenza. Clin Lab Med 2014; 34 (2): 365-85. doi: 10.1016/j.cll.2014.02.009.

15.- Uyeki T M. Influenza diagnosis and treatment in children: a review of studies on clinically useful tests and antiviral treatment for influenza: Pediatr Infect Dis J 2003; 22 (2): 164-77. doi: 10.1097/01.inf.0000050458.35010. b6.

16.- Centers for Disease Control and Prevention (CDC). Information on rapid molecular assays, RT-PCR, and other molecular assays for diagnosis of influenza virus infection. Disponible en: https://www.cdc.gov/flu/ professionals/diagnosis/molecular-assays.htm [citado el 13 de noviembre de 2018].

17.- Chow E J, Mermel L A. Hospital-acquired respiratory viral infections: incidence, morbidity, and mortality in pediatric and adult patients. Open Forum Infect Dis [Internet]. 2017; 4 (1). Disponible en: https://academic. oup.com/ofid/article-lookup/doi/10.1093/ofid/ ofx006 [citado el 31 de agosto de 2017].

18.- Stewardson A J, Sax H, Gayet-Ageron A, Touveneau S, Longtin Y, Zingg W, et al. Enhanced performance feedback and patient participation to improve hand hygiene compliance of health-care workers in the setting of established multimodal promotion: a single-centre, cluster randomised controlled trial. Lancet Infect Dis 2016; 16 (12): 1345-55. doi: 10.1016/S1473-3099(16)30256-0.

19.- Davis R, Parand A, Pinto A, Buetow S. Systematic review of the effectiveness of strategies to encourage patients to remind healthcare professionals about their hand hygiene. J Hosp Infect 2015; 89 (3): 141-62. doi: 10.1016/j.jhin.2014.11.010.

20.- Jiang L, Ng H L, Ho H J, Leo Y S, Prem K, Cook A R, et al. Contacts of healthcare workers, patients and visitors in general wards in Singapore. Epidemiol Infect 2017; 145 (14): 3085-95. doi: 10.1017/S0950268817002035.

21.- Ferguson P E, Jordens C F C, Gilroy N M. Patient and family education in HSCT: improving awareness of respiratory virus infection and influenza vaccination. A descriptive study and brief intervention. Bone Marrow Transplant 2010; 45 (4): 656-61. doi: 10.1038/bmt.2009.209.

22.- Sokol K A, De la Vega-Díaz I, EdmondsonMartin K, Kim S, Tindle S, Wallach F, et al. Masks for prevention of respiratory viruses on the BMT unit: results of a quality initiative. Transpl Infect Dis 2016; 18 (6): 965-7. doi: 10.1111/tid.12608.

23.- Hayward A C. Influenza vaccination of healthcare workers is an important approach for reducing transmission of influenza from staff to vulnerable patients. PLOS ONE. 2017; 12(1): e0169023. https://doi.org/10.1371/journal. pone. 0169023.

24.- Ahmed F, Lindley M C, Allred N, Weinbaum C M, Grohskopf L. Effect of influenza vaccination of healthcare personnel on morbidity and mortality among patients: systematic review and grading of evidence. Clin Infect Dis 2014; 58 (1): 50-7. doi: $10.1093 / \mathrm{cid} / \mathrm{cit} 580$.

25.- Centers for Disease Control and Prevention (CDC) AC on IP. Immunization of health-care personnel: recommendations of the Advisory Committee on Immunization Practices (ACIP). MMWR Recomm Rep 2011; 60 (RR-7): 1-45. PMID: 22108587

26.- Lu P, O'Halloran A C, Ding H, Williams W W, Black C L. Influenza vaccination of healthcare personnel by work setting and occupation-U.S., 2014. Am J Prev Med 2016; 51 (6): 1015-26. doi:10.1016/j.amepre.2016.08.038.

27.- Riedel G. Cobertura vacunacion personal de salud. Rev Chilena Infectol 2017; 34 (3): 294. doi: 10.4067/S0716-10182017000300017.

28.- Pedicino D, Giglio AF, Galiffa VA, Cialdella P, Trotta F, Graziani F, et al. Infections, immunity and atherosclerosis: Pathogenic mechanisms and unsolved questions. Int $\mathrm{J}$ Cardiol. 2013; 166 (3): 572-83. doi: 10.1016/j. ijcard.2012.05.098.

29.- Rezaee-Zavareh M S, Tohidi M, Sabouri A, Ramezani-Binabaj M, Sadeghi-Ghahrodi M, Einollahi B. Infectious and coronary artery disease. ARYA Atheroscler 2016; 12 (1): 41. PMID: 27114736.

30.- Barnes M, Heywood A E, Mahimbo A, Rahman B, Newall AT, Macintyre CR. Acute myocardial infarction and influenza: a meta-analysis of case-control studies. Heart. 2015; 101(21): 1738-47. doi: 10.1136/heartjnl-2015-307691.

31.- Nguyen J L, Yang W, Ito K, Matte T D, Shaman J, Kinney P L. Seasonal influenza infections and cardiovascular disease mortality. JAMA Cardiol 2016; 1 (3): 274. doi: 10.1001/ jamacardio.2016.0433.

32.- Van Lenten B J, Wagner A C, Nayak D P, Hama S, Navab M, Fogelman A M. Highdensity lipoprotein loses its anti-inflammatory properties during acute influenza a infection. Circulation 2001; 103 (18): 2283-8. PMID: 11342478. 
33.- Hebsur S, Vakil E, Oetgen W J, Kumar P N, Lazarous D F. Influenza and coronary artery disease: exploring a clinical association with myocardial infarction and analyzing the utility of vaccination in prevention of myocardial infarction. Rev Cardiovasc Med 2014; 15 (2): 168-75. PMID: 25051134.

34.- Jartti T, Söderlund-Venermo M, Hedman K, Ruuskanen O, Mäkelä M J. New molecular virus detection methods and their clinical value in lower respiratory tract infections in children. Paediatr Respir Rev 2013; 14 (1): 38-45. doi: 10.1016/j.prrv.2012.04.002.

35.- Cawcutt K, Kalil A C. Pneumonia with bacterial and viral coinfection: Curr Opin Crit Care 2017; 23 (5): 385-90. doi: 10.1097/ MCC. 0000000000000435

36.- Loubet P, Voiriot G, Houhou-Fidouh N, Neuville M, Bouadma L, Lescure F-X, et al. Impact of respiratory viruses in hospitalacquired pneumonia in the intensive care unit: A single-center retrospective study. J Clin Virol. 2017; 91: 52-7. doi: 10.1016/j. jcv.2017.04.001.

37.- Kao K-C, Chiu L-C, Hung C-Y, Chang C-H, Yang C-T, Huang C-C, et al. Coinfection and mortality in pneumonia-related acute respiratory distress syndrome patients with bronchoalveolar lavage: a prospective observational study. SHOCK 2017; 47 (5): 61520. doi: $10.1097 /$ SHK.0000000000000802.

38.- Scotta M C, Chakr V C, de Moura A, Becker R G, de Souza A P, Jones M H, et al. Respiratory viral coinfection and disease severity in children: A systematic review and meta-analysis. J Clin Virol. 2016; 80: 45-56. doi: 10.1016/j.jcv.2016.04.019.

39.- Lim F J, de Klerk N, Blyth C C, Fathima P, Moore H C. Systematic review and metaanalysis of respiratory viral coinfections in children: Review on impact of viral coinfection. Respirology 2016; 21 (4): 648-55. doi: $10.1111 /$ resp. 12741
40.- Asner S A, Rose W, Petrich A, Richardson $\mathrm{S}$, Tran D J. Is virus coinfection a predictor of severity in children with viral respiratory infections? Clin Microbiol Infect 2015; 21 (3): 264.e1-6. doi: 10.1016/j.cmi.2014.08.024.

41.- Taylor G, Mitchell R, McGeer A, Frenette C, Suh K N, Wong A, et al. Healthcare-associated influenza in Canadian hospitals from 2006 to 2012. Infect Control Hosp Epidemiol 2014; 35 (2): 169-75. doi: 10.1086/674858.

42.- Instituto de Salud Pública, Chile. Inmunizaciones. Disponible en: http://www. deis.cl/estadisticas-inmunizaciones/ [citado el 12 de noviembre de 2018].

43.- Centers for Disease Control and Prevention (CDC). Efectividad de la vacuna contra la influenza estacional, 2004-2018 [Internet]. Disponible en: https://espanol.cdc.gov/enes/flu/ professionals/vaccination/effectiveness-studies. htm [citado el 12 de noviembre de 2018].

44.- Smetana J, Chlibek R, Shaw J, Splino M, Prymula R. Influenza vaccination in the elderly. Hum Vaccines Immunother. 2018 4; 14 (3): 540-9. doi: 10.1080/21645515.2017.

45.- Jefferson T, Di Pietrantonj C, Al-Ansary L A, Ferroni E, Thorning S, Thomas R E. Vaccines for preventing influenza in the elderly. En: The Cochrane Collaboration, editor. Cochrane Database of Systematic Reviews [Internet]. Chichester, UK: John Wiley \& Sons, Ltd; 2010. Disponible en: http://doi. wiley.com/10.1002/14651858.CD004876.pub3 [citado el 21 de noviembre de 2017].

46.- Mahmud S, Hammond G, Elliott L, Hilderman T, Kurbis C, Caetano P, et al. Effectiveness of the pandemic H1N1 influenza vaccines against laboratory-confirmed H1N1 infections: Population-based case-control study. Vaccine 2011; 29 (45): 7975-81. doi: 10.1016/j. vaccine.2011.08.068.

47.- Osterholm M T, Kelley N S, Sommer A, Belongia E A. Efficacy and effectiveness of influenza vaccines: a systematic review and meta-analysis. Lancet Infect Dis 2012; 12 (1): 36-44. doi: 10.1016/S1473-3099(11)70295-X.

48.- Kelly H, Valenciano M. Estimating the effect of influenza vaccines. Lancet Infect Dis 2012; 12 (1): 5-6. doi: 10.1016/S1473-3099(11)70289-4.

49.- DiazGranados C A, Dunning A J, Kimmel M, Kirby D, Treanor J, Collins A, et al. Efficacy of high-dose versus standard-dose influenza vaccine in older adults. $\mathrm{N}$ Engl J Med 2014; 371(7): 635-45. doi: 10.1056/ NEJMoa1315727.

50.- Seo Y B, Choi W S, Lee J, Song J Y, Cheong H J, Kim W J. Comparison of the immunogenicity and safety of the conventional subunit, MF59adjuvanted, and intradermal influenza vaccines in the elderly. Clin Vaccine Immunol 2014; 21 (7): 989-96. doi: 10.1128/CVI.00615-13.

51.- Weinberger B. Adjuvant strategies to improve vaccination of the elderly population. Curr Opin Pharmacol. 2018; 41: 34-41. doi: 10.1016/j. coph.2018.03.014.

52.- Boonnak K, Dhitavat J, Thantamnu N, Kosoltanapiwat N, Auayporn M, Jiang L, et al. Immune responses to intradermal and intramuscular inactivated influenza vaccine among older age group. Vaccine 2017; 35 (52): 7339-46. doi: 10.1016/j.vaccine.2017.10.106.

53.- Hung I F N, Yuen K-Y. Immunogenicity, safety and tolerability of intradermal influenza vaccines. Hum Vaccines Immunother. 2018; 14 (3): 565-70 doi: 10.1080/21645515.2017.1328332.

54.- Manterola C, Otzen T. Estudios observacionales: los diseños utilizados con mayor frecuencia en investigación clínica. Int J Morphol 2014; 32(2): 634-45. http://dx.doi. org/10.4067/S0717-95022014000200042.

55.- Chacko B, Thomas K, David T, Paul H, Jeyaseelan L, Peter J V. Attributable cost of a nosocomial infection in the intensive care unit: A prospective cohort study. World J Crit Care Med 2017; 6 (1): 79. doi: 10.5492/wjccm. v6.i1.79. 